\title{
Diagnostic criteria for enduring sexual dysfunction after treatment with antidepressants, finasteride and isotretinoin
}

David Healy $^{\mathrm{a}, *}$, Audrey Bahrick ${ }^{\mathrm{b}}$, Maarten Bak $^{\mathrm{c}, \mathrm{d}}$, Angelo Barbato ${ }^{\mathrm{e}}$, Rocco Salvatore Calabrò ${ }^{\mathrm{f}}$, Barbara M. Chubak ${ }^{\mathrm{g}}$, Fiammetta Cosci ${ }^{\mathrm{c}, \mathrm{h}, \mathrm{i}}$, Antonei B. Csoka ${ }^{\mathrm{j}}$, Barbara D’Avanzo $^{\mathrm{e}}$, Silvia Diviccaro ${ }^{\mathrm{k}}$, Silvia Giatti ${ }^{\mathrm{k}}$, Irwin Goldstein ${ }^{1, \mathrm{~m}}$, Heiko Graf $^{\mathrm{n}}$, Wayne J.G. Hellstrom ${ }^{\mathrm{o}}$, Michael S. Irwig ${ }^{\mathrm{p}, \mathrm{q}}$, Emmanuele A. Jannini ${ }^{\mathrm{r}}$, Paddy K.C. Janssen ${ }^{\mathrm{s}, \mathrm{t}}$, Mohit Khera $^{\mathrm{u}}$, Manoj Therayil Kumar ${ }^{\mathrm{v}}$, Joanna Le Noury ${ }^{\mathrm{w}}$, Michał Lew-Starowicz ${ }^{\mathrm{x}}$, David E.J. Linden ${ }^{\mathrm{y}}$, Celine Lüning ${ }^{\mathrm{z}}$, Dee Mangin ${ }^{\mathrm{a}}$, Roberto Cosimo Melcangi ${ }^{\mathrm{k}}$, Omar Walid Muquebil Ali Al Shaban Rodríguez ${ }^{\text {aa }}$, Jalesh N. Panicker ${ }^{\text {ab,ac }}$, Arianna Patacchini ${ }^{\text {h }}$, Amy M. Pearlman ${ }^{\text {ad }}$, Caroline F. Pukall ${ }^{\text {ae }}$, Sanjana Raj ${ }^{\text {af }}$, Yacov Reisman $^{\text {ag }}$, Rachel S. Rubin ${ }^{\text {ah }}$, Rudy Schreiber ${ }^{\text {ai }}$, Stuart Shipko ${ }^{\text {aj }}$, Barbora Vašečkováak and Ahad Waraich ${ }^{\mathrm{m}}$

${ }^{a}$ Department of Family Medicine, McMaster University, Hamilton, ON, Canada

${ }^{\mathrm{b}}$ University Counseling Service, University of Iowa, Iowa City, IA, USA

${ }^{\mathrm{c}}$ Department of Psychiatry and Neuropsychology, Maastricht University, Maastricht, The Netherlands

${ }^{\mathrm{d}}$ FACT, Mondriaan Mental Health, Maastricht/Heerlen, The Netherlands

${ }^{\mathrm{e}}$ Unit for Quality of Care and Rights Promotion in Mental Health, IRCCS Istituto di Ricerche Farmacologiche Mario Negri, Milan, Italy

${ }^{\mathrm{f}}$ IRCCS Centro Neurolesi Bonino-Pulejo, Messina, Italy

${ }^{\mathrm{g}}$ Department of Urology, Icahn School of Medicine at Mount Sinai, New York, NY, USA

${ }^{\mathrm{h}}$ Department of Health Sciences, University of Florence, Florence, Italy

${ }^{\mathrm{i}}$ Clinical Pharmacopsychology Laboratory, University of Florence, Florence, Italy

${ }^{\mathrm{j}}$ Department of Anatomy, Howard University College of Medicine, Washington, DC, USA

${ }^{\mathrm{k}}$ Department of Pharmacological and Biomolecular Sciences, University of Milan, Milan, Italy

${ }^{1}$ Alvarado Hospital, San Diego, CA, USA

${ }^{\mathrm{m}}$ San Diego Sexual Medicine, San Diego, CA, USA

${ }^{\mathrm{n}}$ Department of Psychiatry and Psychotherapy, Ulm University, Ulm, Germany

${ }^{\circ}$ Department of Urology, Tulane University School of Medicine, New Orleans, LA, USA

${ }^{\mathrm{p}}$ Beth Israel Deaconess Medical Center, Boston, MA, USA

${ }^{\mathrm{q}}$ Harvard Medical School, Boston, MA, USA

${ }^{\mathrm{r}}$ Department of Systems Medicine, University of Rome Tor Vergata, Rome, Italy

${ }^{\mathrm{s}}$ Department of Clinical Pharmacy and Toxicology, Maastricht University Medical Centre, Maastricht, The Netherlands

${ }^{\mathrm{t}}$ Department of Hospital Pharmacy, VieCuri Medical Center, Venlo, The Netherlands

"Department of Urology, Baylor School of Medicine, Houston, TX, USA

${ }^{\mathrm{v}}$ Institute for Mind and Brain, Kerala, India 
${ }^{\mathrm{w}}$ North Wales Department of Psychological Medicine, Bangor, Wales, UK

${ }^{\mathrm{x}}$ Department of Psychiatry, Centre of Postgraduate Medical Education, Warsaw, Poland

${ }^{\mathrm{y}}$ Department of Psychiatry and Neuropsychology, Faculty of Health, Medicine and Life Sciences, School for Mental Health and Neuroscience, Maastricht University, Maastricht, The Netherlands ${ }^{\mathrm{z}}$ Department of Psychology and Psychotherapy, Witten/Herdecke University, Witten, Germany

${ }^{a}$ Psychiatry Service, Hospital Universitario San Agustín, Avilés, Asturias, Spain

${ }^{\text {ab }}$ Department of Uro-Neurology, The National Hospital for Neurology and Neurosurgery, Queen Square, London, UK

${ }^{\mathrm{ac}}$ Department of Brain Repair and Rehabilitation, UCL Queen Square Institute of Neurology, Faculty of Brain Sciences, University College London, London, UK

${ }^{\text {ad }}$ Carver College of Medicine, University of Iowa Health Care, Iowa City, IA, USA

${ }^{a e}$ Department of Psychology, Queen's University, Kingston, ON, Canada

${ }^{\text {af }}$ Faculty of Social and Behavioural Sciences, Utrecht University, Utrecht, The Netherlands

${ }^{a g}$ Flare-Health, Amstelveen, The Netherlands

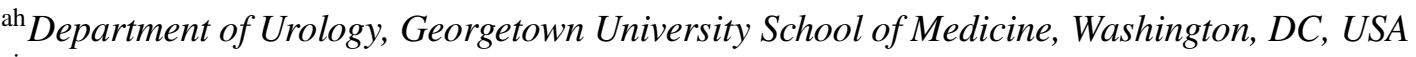

${ }^{a i}$ Department of Neuropsychology and Psychopharmacology, Faculty of Psychology and Neuroscience, Maastricht University, Maastricht, The Netherlands

aj Private Practice, Pasadena, CA, USA

${ }^{\mathrm{k}}$ Psychiatric Clinic, Slovak Medical University and University Hospital, Bratislava, Slovakia

Received 30 April 2021

Accepted 7 October 2021

\begin{abstract}
.
BACKGROUND: A set of enduring conditions have been reported in the literature involving persistent sexual dysfunction after discontinuation of serotonin reuptake inhibiting antidepressants, 5 alpha-reductase inhibitors and isotretinoin.

OBJECTIVE: To develop diagnostic criteria for post-SSRI sexual dysfunction (PSSD), persistent genital arousal disorder (PGAD) following serotonin reuptake inhibitors, post-finasteride syndrome (PFS) and post-retinoid sexual dysfunction (PRSD). METHODS: The original draft was designed using data from two published case series (Hogan et al., 2014 and Healy et al., 2018), which represent the largest public collections of data on these enduring conditions. It was further developed with the involvement of a multidisciplinary panel of experts.

RESULTS: A set of criteria were agreed upon for each of the above conditions. Features of PSSD, PFS and PRSD commonly include decreased genital and orgasmic sensation, decreased sexual desire and erectile dysfunction. Ancillary non-sexual symptoms vary depending on the specific condition but can include emotional blunting and cognitive impairment. PGAD presents with an almost mirror image of unwanted sensations of genital arousal or irritability in the absence of sexual desire. A new term, post-SSRI asexuality, is introduced to describe a dampening of sexual interest and pleasure resulting from a pre-natal or pre-teen exposure to a serotonin reuptake inhibitor.

CONCLUSIONS: These criteria will help in both clinical and research settings. As with all criteria, they will likely need modification in the light of developments.
\end{abstract}

Keywords: Post-SSRI sexual dysfunction, antidepressants, selective serotonin reuptake inhibitors, finasteride, isotretinoin

\footnotetext{
*Address for correspondence: David Healy, Department of Family Medicine, David Braley Health Sciences Centre, McMaster University, 100 Main Street West, 5th Floor, Hamilton, ON L8P 1H6, Canada. Tel.: +1 365336 9817; E-mail: david.healy54@gmail.com.
} 


\section{Introduction}

There is a growing awareness that a substantial number of medicines have effects, some positive and some negative, on sexual functioning. These include antibiotics, anti-hypertensives, antidepressants, lipid lowering agents, medicines affecting endocrine systems and others [1].

There is a set of effects that endure after treatment stops that at present are most closely linked to serotonin reuptake inhibiting drugs, 5 alpha-reductase inhibitors, and isotretinoin. These include persistent genital arousal disorder (PGAD) first formally described in 2001 [2], post-SSRI sexual dysfunction (PSSD) described in 2006 [3,4], and post-finasteride syndrome (PFS) described in 2011 [5]. Cases of persistent sexual dysfunction following isotretinoin were reported in this journal in 2014 [6], with the condition designated as a post-retinoid sexual dysfunction (PRSD) in 2018 [1]. For each of these conditions, it is now clear that cases had been reported or were present for a decade or more before appearing in the academic literature. The Medicines and Healthcare products Regulatory Agency (MHRA) have indicated that their first reports of a sexual dysfunction that continued after an SSRI was withdrawn involved fluvoxamine in 1987 and fluoxetine in 1991 [7].

The incidence of these conditions is unknown. The number of patients who regain $100 \%$ of their original sexual function after stopping serotonin reuptake inhibitors, 5 alpha-reductase inhibitors and isotretinoin has never been properly investigated. Clinical trials do not routinely include follow-up to assess the resolution of adverse sexual effects, and it would be unethical to carry out a randomized controlled trial which specifically intended to produce an enduring sexual dysfunction in participants in order to establish prevalence. Several healthy volunteer trials involving SSRIs that included follow-up have hinted at incidence rates of enduring changes in sexual functioning following discontinuation [8]. In one clinical study, $55 \%$ of participants still had sexual dysfunction six months after switching from an SSRI to amineptine compared to $4 \%$ who were treated solely with amineptine, a drug with no action on the serotonin system [9]. Three large studies into the use of SSRIs for premature ejaculation reported that the ejaculation-delaying effects of the medication persisted for a significant number of participants at 3and 6-month follow-up after withdrawal of the drug [10-12]. A healthy volunteer study which assessed the effects of paroxetine on sperm and sexual function reported that brief sexual function inventory (BSFI) scores for erectile and ejaculatory functions had not returned to baseline four weeks after discontinuation of the drug, with $9 \%$ of patients complaining of more than mild dysfunction [13].

While the ability to diagnose adverse effects should be part of any prescriber's skill set, the fact that these conditions can persist or emerge upon stopping the causative drug adds an extra level of complexity. In addition, these conditions are little known and poorly understood by healthcare professionals.

Neglecting these disorders risks further harm to patients in terms of dismissive responses from clinicians, unnecessary investigations, and misdiagnosis as a psychogenic problem which may result in further use of the causative drug. A qualitative study into the experiences of PSSD patients when engaging with healthcare professionals highlighted the difficulties that patients can face when trying to seek help [14].

In the absence of a biomarker, diagnostic criteria may improve both research and clinical practice. The criteria below offer a template for researchers, clinicians, patients, regulators and pharmaceutical companies to support communication. They will hopefully increase the precision of phenotyping leading to a more accurate quantification of the burden of these problems and will provide an operational framework to facilitate multicentric collaborative research. 


\section{Methods}

The 2014 and 2018 case series from Hogan et al. and Healy et al. represent the largest public collections of data on these enduring conditions and were used as a starting point for the original draft $[1,6]$. Common features were identified and used to formulate a preliminary set of criteria. The data for those papers were sourced from RxISK.org, an independent drug safety website that features a global adverse event reporting facility. RxISK currently has around 1000 reports of these conditions.

A multidisciplinary panel of experts was enlisted to further develop the article. Many of the group members are authors of peer reviewed literature on these conditions. Several have successfully petitioned regulators in North America and Europe on the issue of PSSD, resulting in warnings about persistent sexual dysfunction after discontinuation of treatment being added to medication labels [15].

The original draft was circulated to the group who made amendments based on published data, clinical and research experience, and assessment of criteria suitability across international clinical settings. Successive drafts were modified in response to comments and reissued until agreement was reached.

\section{Post-SSRI sexual dysfunction (PSSD)}

Significant sexual dysfunction can happen while on treatment and after stopping any serotonin reuptake inhibiting (SRI) drug [1,3,4,6,14-20]. SRIs include the selective serotonin reuptake inhibitors (SSRIs), serotonin-norepinephrine reuptake inhibitors (SNRIs), SRI tricyclic antidepressants, SRI antihistamines, tetracycline antibiotics such as doxycycline, and analgesics like tramadol.

PSSD here refers to a common syndrome happening after any SRI. It is independent of any pre-existing or reactive mental health problem and can be triggered in healthy volunteers after exposures of a few weeks.

\section{Criteria:}

\section{Necessary}

(1) Prior treatment with a serotonin reuptake inhibitor.

(2) An enduring change in somatic (tactile) or erogenous (sexual) genital sensation after treatment stops.

Additional

(3) Enduring reduction or loss of sexual desire.

(4) Enduring erectile dysfunction (males).

(5) Enduring inability to orgasm or decreased sensation of pleasure during orgasm.

(6) The problem is present for $\geq 3$ months after stopping treatment.

There should be

(7) No evidence of pre-drug sexual dysfunction that matches the current profile.

(8) No current medical conditions that could account for the symptoms.

(9) No current medication or substance misuse that could account for the symptoms.

Ancillary sexual symptoms may include:

- genital pain

- reduced nipple sensitivity

- decreased or loss of nocturnal erections (males)

- reduced ejaculatory force (males) 
- flaccid glans during erection (males)

- decreased vaginal lubrication (females).

Ancillary non-sexual symptoms may include:

- emotional numbing

- depersonalization

- other sensory problems involving skin, smell, taste or vision $[1,3,21]$

- cognitive impairment.

Close to all people taking an SRI have some alteration of genital sensitivity within an hour of starting treatment. This may be noticeable or obscured by an apparent benefit such as improvement in premature ejaculation in men.

In PSSD there is a marked escalation of genital sensory effects [22,23]. Some describe reduced somatic (tactile) sensation - genitals feel like they were exposed to an anesthetic. Others describe reduced erogenous (sexual) sensation - genital touch feels like being touched on any other body part. The condition may be triggered by exposures as brief as several days [1].

Changes in genital sensation may be hidden beneath symptoms of loss of sexual desire or arousal. It is important to enquire about changes in tactile and erogenous genital sensation, as well as changes in orgasm sensation, as these sensory changes seem distinctive to PSSD. Making the diagnosis of PSSD rather than another form of enduring sexual dysfunction depends on the presence of these symptoms.

PSSD resembles tardive dyskinesia which is linked to withdrawal of antipsychotics but can be present prior to withdrawal. Where an individual cannot stop their SRI, a provisional diagnosis might be warranted.

The sexual dysfunction that accompanies normal antidepressant use may linger for some days or weeks following discontinuation of treatment. The present consensus is that once dysfunction lasts for three months, it is more likely to be PSSD. In the case of standard withdrawal syndromes, there is no indication that patients could rub a hard bristled brush up and down their genitals and not feel it, as has been described for PSSD, although not all PSSD patients are as badly affected as this.

PSSD can vary in severity. Sexual side effects while taking an antidepressant can sometimes improve significantly upon stopping the drug but still leave residual symptoms. For example, a patient may regain the ability to achieve orgasm upon stopping the antidepressant after being completely unable to do so while on treatment, but the orgasm no longer feels the same as pre-drug. Regardless of whether persisting sexual effects are severe or mild, they should be classified as PSSD if there hasn't been a full return to pre-drug baseline.

Emotional and genital numbing commonly go together. But emotional numbing also happens on antipsychotics without sexual dysfunction of this sort, and marked depersonalization can happen on drugs that do not cause sexual dysfunction. Emotional numbing or depersonalization may be a person's main complaint in primary care, but this is less likely in a sexual dysfunction clinic.

In a 2018 study, two subjects reported penile curvature as part of PSSD [1]. Results of a ten-year retrospective review of clinical PSSD cases, published in 2020, provided further evidence that PSSD may result in damage to the erectile tissue of the penis [24]. This seems to overlap with findings from a major PFS study [25]. The discovery of erectile tissue abnormalities on an ultrasound scan might therefore support rather than preclude a diagnosis of PSSD. Whether these changes are part of the syndrome or co-incidental is uncertain. 
There are reports of some patients experiencing a brief improvement in their PSSD linked to stopping another drug where their condition lifts for a few days only to return afterwards. However, this has failed to lead to any treatment options.

PSSD is sometimes accompanied by symptoms of other pelvic conditions such as:

- Pelvic floor dysfunction in men or women.

- Interstitial cystitis/painful bladder syndrome in women, often diagnosed as recurrent urinary tract infections.

- Interstitial cystitis/painful bladder syndrome in men, often diagnosed as recurrent prostatitis.

- Change in seminal volume and/or quality in men (this also happens while on SRI treatment).

SRIs can cause these conditions with or without sexual dysfunction. They risk further investigations and potentially unhelpful, even damaging, treatment if a link to prior SRI or related treatment (where present) is not recognised.

\section{Persistent genital arousal disorder (PGAD)}

PGAD can occur after trauma to the genital or pelvic area, may accompany Tarlov cysts, interstitial cystitis, or other pelvic conditions, seems more common perimenopausally and postmenopausally, and has been linked to a number of medicines that do not have effects on the serotonin system [26].

However, one of its commoner triggers is stopping serotonin reuptake inhibiting (SRI) drugs [1,27-30]. PGAD happening after SRIs can appear like a mirror image of PSSD with intense and painful or abnormal genital sensations and spontaneous orgasms.

PGAD is commonly seen in pain clinics. Women may have a history of desperate remedies tried, and many will be members of online support forums. The treatment history is likely to include gabapentinoids, SSRIs for their genital anesthetic effect, opioids, botox, and more drastic interventions up to clitoridectomy and pudendal nerve ablation.

It is not clear if men are affected, but cases of premature or spontaneous ejaculation after discontinuation of an SRI have been reported [1,31]. Some cases of PSSD may show alternating arousal (PGAD) or dysfunction (PSSD), and some may evolve into PGAD or vice versa.

In psychosexual therapy settings, the question of links to sexual trauma/abuse is present. Clearly, sexual abuse is common and some people with PGAD may have a background of sexual trauma, but at present the two states do not appear linked.

\section{Criteria:}

\section{Necessary}

(1) Persistent unwelcome sensations of genital arousal or irritability.

(2) The genital arousal is painful or altered in quality rather than pleasurable.

(3) Sexual activity provides limited or no resolution.

(4) The abnormal sensations can be triggered by non-sexual stimuli.

Additional

(5) Spontaneous unwelcome and unpleasant orgasmic events.

(6) The condition is highly distressing and ego-dystonic.

(7) Management strategies such as access to icepacks are necessary.

(8) A history of efforts to have the problem treated is common. 
(9) The problem is continuous (although episodic exacerbations/remissions may occur).

(10) If linked to an SRI, the problem starts on discontinuation of the drug.

(11) The problem is present for $\geq 3$ months after stopping treatment.

Ancillary conditions

- Interstitial cystitis/painful bladder syndrome

- Restless legs syndrome

- Pelvic floor dysfunction.

More detailed information about PGAD is available in a recently published consensus article by the International Society for the Study of Women's Sexual Health (ISSWSH) [26].

\section{Post-finasteride syndrome (PFS)}

Finasteride, dutasteride and saw palmetto are 5 alpha-reductase inhibitors that can trigger an enduring sexual dysfunction with a very similar profile to PSSD [32]. As the name suggests, PFS is most commonly associated with finasteride which was licensed for the treatment of male pattern baldness in 1997. In 2011, a warning for erectile dysfunction that persisted after stopping treatment was added to the US product label for the finasteride products, Propecia and Proscar. This was updated in 2012 to include libido disorders, ejaculation disorders and orgasm disorders that continued after discontinuation of Propecia, and decreased libido that continued after discontinuation of Proscar [33]. There should be no prior use of isotretinoin or an SRI when making a PFS diagnosis. Sexual dysfunction that happens on finasteride but clears after treatment stops is not PFS.

\section{Criteria:}

Necessary

(1) Prior treatment with a 5 alpha-reductase inhibitor.

(2) Enduring sexual dysfunction after stopping treatment.

\section{Additional}

(3) Enduring reduction or loss of sexual desire.

(4) Enduring erectile dysfunction.

(5) Enduring reduction in genital and orgasmic sensation.

(6) The problem is present for $\geq 3$ months after stopping treatment.

There should be

(7) No evidence of pre-drug sexual dysfunction that matches the current profile.

(8) No current medical conditions that could account for the symptoms.

(9) No current medication or substance misuse that could account for the symptoms.

(10) No other prior medication that could account for the symptoms.

A number of related features may stem from finasteride without being diagnostic for PFS:

- gynecomastia

- altered seminal quantity and quality.

Additional finasteride effects that can occur independently of any sexual difficulties but may also accompany sexual problems include: 
- cognitive impairment

- depression

- suicidality.

There is some evidence for penile curvature or other penile effects in men with PFS [25], but it is not clear if this was present prior to treatment or has resulted since. Alterations in neuroactive steroid levels [34], methylation of 5 alpha-reductase type 2 in cerebrospinal fluid [35], and gut microbiota population [36] have also been found in PFS patients. But again, it is not clear whether these were present before treatment.

\section{Post-retinoid sexual dysfunction (PRSD)}

Isotretinoin is a retinoid medication used in the treatment of acne and has been linked to sexual dysfunction in the literature [37,38]. In 2017, Health Canada and the European Medicines Agency recommended that a warning for erectile dysfunction should be added to the product information for isotretinoin products, with decreased libido additionally recommended for inclusion in European labels $[39,40]$.

Reports of an enduring sexual dysfunction with a similar profile to PSSD and PFS were published in 2014 [6] and 2018 [1]. The Netherlands Pharmacovigilance Centre Lareb reported seven cases of sexual dysfunction linked to isotretinoin, three of which had not resolved after stopping [41].

Isotretinoin has both serotonin reuptake inhibiting and 5 alpha-reductase inhibiting properties, but it is not clear whether the problem stems from either of these actions. There should be no prior use of finasteride or an SRI when making a PRSD diagnosis. There may be some sexual dysfunction on treatment, but it is not clear what proportion of these problems persist after treatment stops.

\section{Criteria:}

\section{Necessary}

(1) Prior treatment with isotretinoin.

(2) Enduring sexual dysfunction after stopping treatment.

\section{Additional}

(3) Enduring reduction or loss of sexual desire.

(4) Enduring erectile dysfunction (males) or loss of vaginal lubrication (females).

(5) Enduring reduction in genital and orgasmic sensation.

(6) The problem is present for $\geq 3$ months after stopping treatment.

There should be

(7) No evidence of pre-drug sexual dysfunction that matches the current profile.

(8) No current medical conditions that could account for the symptoms.

(9) No current medication or substance misuse that could account for the symptoms.

(10) No other prior medication that could account for the symptoms.

A number of related features may stem from isotretinoin without being diagnostic for PRSD eg. gynecomastia.

Additional isotretinoin effects that can occur independently of any sexual difficulties but may also accompany sexual problems include: 
- cognitive impairment

- skin changes

- joint changes

- bowel changes

- depression

- suicidality.

The term Post-Accutane Syndrome (PAS) is in widespread use and often refers to what is termed PRSD here but equally often appears to include suicidality and other cognitive effects without sexual difficulties.

\section{Post-SSRI asexuality}

Animal studies have found that exposure to an SSRI during pregnancy or at an early age produces sexual deficits in adulthood [42]. A recent study in humans found evidence that there may be similar implications for people exposed to an SSRI in childhood [43]. Given that these problems develop prior to sexual maturity, the person may have no pre-drug baseline for comparison, making it difficult to recognise symptoms such as low sexual desire, reduced genital sensation and pleasureless orgasm. We propose the introduction of a new term, Post-SSRI asexuality, to describe a dampening of sexual interest and pleasure resulting from a pre-natal or pre-teen exposure to a serotonin reuptake inhibitor. At present there are no data on the proportion of people who define themselves as asexual and have a condition that may be linked to medications.

\section{Criteria:}

(1) Clear evidence of maternal SRI intake in pregnancy or

(2) Evidence of extended pre-teen SRI intake.

(3) Lack of sexual interest in either the same sex or the opposite sex.

(4) No experience of pleasure from masturbation or sexual activity ever.

(5) Identification as asexual.

\section{Other drugs}

There are reports of a persistent sexual dysfunction that resembles PSSD after other psychotropic drugs that are not SRIs including mirtazapine and aripiprazole. There is insufficient data to know whether these states form a related or different syndrome.

Novel oral anticoagulants (NOACs) like rivaroxaban and dabigatran also appear to have produced clinical pictures that resemble PSSD and PFS. There are online reports but no peer reviewed literature about this.

In contrast, antipsychotics cause sexual difficulties including a loss of sexual desire, erectile dysfunction, retrograde ejaculation and other problems. These states may endure, but they are not PSSD.

Norepinephrine reuptake inhibitors produce erectile dysfunction which may persist in some instances. This is not PSSD.

A range of other drugs such as thiazide antihypertensives cause sexual dysfunction and some of these might endure, but at present there is no evidence that these are the same as PSSD or PFS. 


\section{Diagnostic interviewing}

The criteria are intended for use alongside diagnostic interviewing which should involve a detailed drug history. SRIs, 5 alpha-reductase inhibitors and isotretinoin may cause an enduring sexual dysfunction even though prior use of the same drug or another from the same class did not produce a significant problem. Patients with lifelong sexual dysfunction without prior use of SRIs, 5 alpha-reductase inhibitors or isotretinoin, and without any obvious explanation for the problem, should be asked about possible pre-natal exposure to an SRI.

The challenge-dechallenge-rechallenge strategy which is sometimes used to assess whether an effect is linked to a medication is not suitable for investigating adverse effects that persist or worsen after a medication is stopped.

Patients with PSSD, PFS, PRSD or PGAD typically have normal sexual functioning prior to starting treatment. If a patient had prior sexual difficulties, it is important to decide on the degree of overlap to the current problem. A current diagnosis of a mental health disorder does not prevent a diagnosis of these enduring conditions. No psychiatric condition causes genital numbness or irritability to the point that clitoridectomy might be contemplated.

These enduring conditions can remain for months, years or indefinitely after the causative drug is stopped. There are reports of PSSD and PRSD having persisted for over two decades following discontinuation of the drug [1], The criteria therefore provide no upper limit in terms of duration.

In some cases, an enduring sexual dysfunction may only appear when the drug is stopped, or a mild dysfunction on treatment may worsen on stopping, sometimes with the appearance of new features. For example, in the case of SSRIs, premature ejaculation seems to be most closely associated with stopping the drug. There is a suggestion that a delay can sometimes occur between the last dose of the drug and the onset of dysfunction ie. typically up to a couple of weeks. At present, as the data on this is limited, it is not possible to set a time limit after which an enduring condition cannot be diagnosed, particularly if all criteria are met and the clinical presentation is otherwise textbook. A degree of discretion may be warranted in such cases when assessing a possible link to the medication.

PSSD, PFS and PRSD are sometimes linked to borderline testosterone or other hormone levels. This may be a consequence of the conditions rather than a cause. Testosterone treatment has not been reported to reliably benefit these syndromes. Moreover, the efficacy of phosphodiesterase type 5 (PDE5) inhibitors appears reduced in these conditions.

If available, quantitative sensory testing (QST) of the penis (vibration, warm and cold temperatures) may be helpful in assessing somatic sensation [24]. Erogenous sensation is poorly understood and there are no available tests.

\section{Conclusion}

We hope these criteria will lead to increased awareness of the conditions, more accurate diagnoses, and greater research efforts into treatment options for those affected. As with all criteria, they will likely need modification in the light of developments. It is hoped they will ultimately be replaced by diagnostic markers, whether genetic, electrophysiological, endocrine or other. 


\section{Acknowledgements}

Emmanuele A. Jannini is supported in part by the Italian Ministry of University and Research Grant PRIN \#2017S9KTNE_002. Dee Mangin is supported by the David Braley Chair in Family Medicine, McMaster University. Jalesh N. Panicker is supported in part by funding from the United Kingdom's Department of Health NIHR Biomedical Research Centres.

\section{Conflict of interest}

David Healy, Joanna Le Noury and Dee Mangin are linked to RxISK.org which records patient reported adverse events on drugs. The website has featured information about enduring sexual dysfunctions after the use of antidepressants and other drugs.

\section{Funding}

None to report.

\section{References}

[1] Healy D, Le Noury J, Mangin D. Enduring sexual dysfunction after treatment with antidepressants, $5 \alpha$-reductase inhibitors and isotretinoin: 300 cases. Int J Risk Saf Med. 2018;29(3-4):125-34.

[2] Leiblum SR, Nathan SG. Persistent sexual arousal syndrome. A newly discovered pattern of female sexuality. J Sex Marital Ther. 2001;27(4):365-80.

[3] Csoka AB, Shipko S. Persistent sexual side effects after SSRI discontinuation. Psychother Psychosom. 2006;75(3):187-8.

[4] Bahrick AS. Post SSRI sexual dysfunction. American Society for the Advancement of Pharmacotherapy Tablet. 2006;7(3):2-3, 10-11.

[5] Irwig MS, Kolukula S. Persistent sexual side effects of finasteride for male pattern hair loss. J Sex Med. 2011;8(6):1747-53.

[6] Hogan C, Le Noury J, Healy D, Mangin D. One hundred and twenty cases of enduring sexual dysfunction following treatment. Int J Risk Saf Med. 2014;26(2):109-16.

[7] Communication from MHRA to Kevin Bennett, forwarded to David Healy.

[8] SmithKline Beecham. 1986. HP/85/33/A. A study of paroxetine pharmacokinetics.

[9] Montejo AL, Llorca G, Izquierdo JA, Carrasco JL, Daniel E, Pérez-Sola V et al. Disfunción sexual con antidepresivos. Efecto del cambio a amineptino en pacientes con disfunción sexual secundaria a ISRS [Sexual dysfunction with antidepressive agents. Effect of the change to amineptine in patients with sexual dysfunction secondary to SSRI]. Actas Esp Psiquiatr. 1999;27(1):23-34.

[10] Safarinejad MR, Hosseini SY. Safety and efficacy of citalopram in the treatment of premature ejaculation: A double-blind placebo-controlled, fixed dose, randomized study. Int J Impot Res. 2006;18(2):164-9.

[11] Arafa M, Shamloul R. Efficacy of sertraline hydrochloride in treatment of premature ejaculation: A placebo-controlled study using a validated questionnaire. Int J Impot Res. 2006;18(6):534-8.

[12] Safarinejad MR. Safety and efficacy of escitalopram in the treatment of premature ejaculation: A double-blind, placebocontrolled, fixed-dose, randomized study. J Clin Psychopharmacol. 2007;27(5):444-50.

[13] Tanrikut C, Feldman AS, Altemus M, Paduch DA, Schlegel PN. Adverse effect of paroxetine on sperm. Fertil Steril. 2010;94(3):1021-6.

[14] Healy D, Le Noury J, Mangin D. Post-SSRI sexual dysfunction: Patient experiences of engagement with healthcare professionals. Int J Risk Saf Med. 2019;30(3):167-78.

[15] Healy D. Citizen petition: Sexual side effects of SSRIs and SNRIs. Int J Risk Saf Med. 2018;29(3-4):135-47.

[16] Csoka AB, Bahrick A, Mehtonen OP. Persistent sexual dysfunction after discontinuation of selective serotonin reuptake inhibitors. J Sex Med. 2008;5(1):227-33.

[17] The Netherlands Pharmacovigilance Centre Lareb. SSRIs and persistent sexual dysfunction. 2012. Available from: https://databankws.lareb.nl/downloads/KWB_2012_3_SSRI.pdf. 
[18] Muquebil Ali Al Shaban Rodríguez OW, Álvarez de Morales Gómez-Moreno E, Fernández Fernández J, Fresno García C, del Mar Fernández Fernández M. Disfunción sexual persistente tras el tratamiento con inhibidores selectivos de la recaptación de serotonina: A propósito de un caso tras la retirada de paroxetina. Psiquiatría Biológica. 2017;24(2):70-2.

[19] Calabrò RS, De Luca R, Manuli A, Portaro S, Naro A, Quattrini F. Towards improving post-SSRI sexual dysfunction by using nutriceuticals: Lessons from a case study. J Sex Marital Ther. 2019;45(6):562-5.

[20] Reisman Y. Post-SSRI sexual dysfunction. BMJ. 2020;368:m754.

[21] Healy D, Mangin D, Lochhead J. Development and persistence of patient-reported visual problems associated with serotonin reuptake inhibiting antidepressants. Int J Risk Saf Med. 2021. doi:10.3233/JRS-210018.

[22] Kauffman RP, Murdock A. Prolonged post-treatment genital anesthesia and sexual dysfunction following discontinuation of citalopram and the atypical antidepressant nefazodone. The Open Women' Health Journal. 2007;1:1-3.

[23] Waldinger MD, van Coevorden RS, Schweitzer DH, Georgiadis J. Penile anesthesia in post SSRI sexual dysfunction (PSSD) responds to low-power laser irradiation: A case study and hypothesis about the role of transient receptor potential (TRP) ion channels. Eur J Pharmacol. 2015;753:263-8.

[24] Waraich A, Clemons C, Ramirez R, Yih J, Goldstein S, Goldstein I. Post-SSRI sexual dysfunction (PSSD): Ten year retrospective chart review. J Urol. 2020;203:e1179.

[25] Khera M, Than JK, Anaissie J, Antar A, Song W, Losso B et al. Penile vascular abnormalities in young men with persistent side effects after finasteride use for the treatment of androgenic alopecia. Transl Androl Urol. 2020;9(3):1201-9.

[26] Goldstein I, Komisaruk BR, Pukall CF, Kim NN, Goldstein AT, Goldstein SW et al. International society for the study of Women's sexual health (ISSWSH) review of epidemiology and pathophysiology, and a consensus nomenclature and process of care for the management of persistent genital arousal disorder/genito-pelvic dysesthesia (PGAD/GPD). J Sex Med. 2021;18(4):665-97.

[27] Freed L. Persistent sexual arousal syndrome. J Sex Med. 2005;2:743.

[28] Leiblum SR, Goldmeier D. Persistent genital arousal disorder in women: Case reports of association with anti-depressant usage and withdrawal. J Sex Marital Ther. 2008;34(2):150-9.

[29] Eibye S, Jensen HM. Persistent genital arousal disorder: Confluent patient history of agitated depression, paroxetine cessation, and a tarlov cyst. Case Rep Psychiatry. 2014;2014:529052.

[30] de Magalhães FJ, Kumar MT. Persistent genital arousal disorder following selective serotonin reuptake inhibitor cessation. J Clin Psychopharmacol. 2015;35(3):352-4.

[31] Adson DE, Kotlyar M. Premature ejaculation associated with citalopram withdrawal. Ann Pharmacother. 2003;37(12):1804-6.

[32] Giatti S, Diviccaro S, Panzica G, Melcangi RC. Post-finasteride syndrome and post-SSRI sexual dysfunction: two sides of the same coin? Endocrine. 2018;61(2):180-93.

[33] FDA. Questions and answers: Finasteride label changes. Available from: https://wayback.archive-it.org/7993/2017072 3090425/https://www.fda.gov/Drugs/DrugSafety/InformationbyDrugClass/ucm299754.htm.

[34] Melcangi RC, Santi D, Spezzano R, Grimoldi M, Tabacchi T, Fusco ML et al. Neuroactive steroid levels and psychiatric and andrological features in post-finasteride patients. J Steroid Biochem Mol Biol. 2017;171:229-35.

[35] Melcangi RC, Casarini L, Marino M, Santi D, Sperduti S, Giatti S et al. Altered methylation pattern of the SRD5A2 gene in the cerebrospinal fluid of post-finasteride patients: A pilot study. Endocr Connect. 2019;8(8):1118-25.

[36] Borgo F, Macandog AD, Diviccaro S, Falvo E, Giatti S, Cavaletti G et al. Alterations of gut microbiota composition in post-finasteride patients: A pilot study. J Endocrinol Invest. 2021;44(6):1263-73.

[37] Coleman R, MacDonald D. Effects of isotretinoin on male reproductive system. Lancet. 1994;344(8916)198.

[38] Tirado Sánchez A, León Dorantes G. Disfunción eréctil durante el tratamiento con isotretinoína [Erectile dysfunction during isotretinoin therapy]. Actas Urol Esp. 2005;29(10):974-6.

[39] Health Canada. Summary safety review - oral retinoid products - assessing the potential risk of impotence (erectile dysfunction). Available from: https://www.canada.ca/en/health-canada/services/drugs-health-products/medeffectcanada/safety-reviews/summary-safety-review-oral-retinoid-products-assessing-potential-risk-erectile-dysfunction.html.

[40] GOV.UK. Isotretinoin (Roaccutane): rare reports of erectile dysfunction and decreased libido. Available from: https://www. gov.uk/drug-safety-update/isotretinoin-roaccutane-rare-reports-of-erectile-dysfunction-and-decreased-libido.

[41] The Netherlands Pharmacovigilance Centre Lareb. Isotretinoin and erectile dysfunction. 2015. Available from: https://databankws.lareb.nl/Downloads/KWB_2015_2_isotr.pdf.

[42] Simonsen AL, Danborg PB, Gøtzsche PC. Persistent sexual dysfunction after early exposure to SSRIs: Systematic review of animal studies. Int J Risk Saf Med. 2016;28(1):1-12.

[43] Lorenz TK. Antidepressant use during development may impair Women's sexual desire in adulthood. J Sex Med. 2020;17(3):470-6. 